\title{
Changes in grapevine berry skin photochemistry may support metabolic responses to postharvest treatment by ultraviolet light
}

\author{
K. CSEPREGI*, P. TESZLÁK ${ }^{* *}$, A. RÁCZ*, Gy. CZÉGÉNY*, L. KŐRÖSI**, and É. HIDEG*,+ \\ Department of Plant Biology, Faculty of Sciences, University of Pécs, Ifjúság u. 6., Pécs 7624, Hungary* \\ Research Institute for Viticulture and Oenology, University of Pécs, Pázmány Péter u. 4., Pécs 7634, Hungary**
}

\begin{abstract}
Harvested mature berry clusters of two white table grape cultivars were used to study the effects of postharvest UV irradiation. One cultivar, 'Queen of Vineyard' (QV), had higher light-acclimated PSII quantum yield, higher phenolic contents, and stronger total antioxidant capacities than the other, 'White Sultana' (WS). These differences were maintained throughout the experiment. Responses of the two cultivars to a 30-min UV irradiation were also different. Antioxidant capacities and flavonol, especially quercetin-3-O-glucuronide, contents were lower $2 \mathrm{~h}$ after the UV treatment in both cultivars and recovered in QV but not in WS berry skins later on. Our data demonstrate that mature grapevine berries have photosynthetically active tissues capable of dynamic changes even several hours after harvest and suggest that changes in photochemistry may contribute to postharvest metabolic responses of berry skins. Results also support the potential of postharvest manipulation of fruit qualities with UV irradiation.
\end{abstract}

Keywords: nonphotochemical quenching; polyphenols; total antioxidant capacity; ultraviolet absorbance.

\section{Introduction}

Grapevine (Vitis vinifera L.) berries are important source of flavonoids (Conde et al. 2007) with high nutraceutical values due to their antioxidant capacities (Graf et al. 2005). Flavonoids, as well as other plant polyphenols are synthesized via the phenylpropanoid pathway in the skin of berries (Braidot et al. 2008); their biosynthesis is transcriptionally regulated by UV-B $(280-315 \mathrm{~nm})$ radiation (Carbonell-Bejerano et al. 2014). These regulatory pathways remain active in detached fruits and postharvest UV treatments of berry clusters have been shown to affect berry skin phenolic contents either positively (Cantos et al. 2000, Sheng et al. 2018) or negatively (Csepregi et al. 2019). In a recent study, using a thick-skinned red table grape 'Emperor', we suggested

\section{Highlights}

- Postharvest UV irradiation affects grapevine berry skin photochemistry

- The cultivar with higher photochemical yield was more UV tolerant

- Berry skin photosynthesis may support changes in antioxidative metabolites
Received 9 April 2021

Accepted 19 April 2021

Published online 5 May 2021

${ }^{+}$Corresponding author

phone: +36-72-503-600

e-mail: ehideg@gamma.ttk.pte.hu

Abbreviations: ABTS - 2,2'-azino-bis(3-ethylbenzothiazoline)-6-sulfonatic acid; CA - caftaric acid; DM - dry mass; $\mathrm{F}_{0}$ - minimal fluorescence yield of the dark-adapted state; $F_{m}$ - maximal fluorescence yield of the dark-adapted state; $F_{m}{ }^{\prime}-$ maximal fluorescence yield of the light-adapted state; FRAP - ferric reducing antioxidant potential; $F_{v}$ - variable fluorescence; $F_{v} / F_{m}-$ maximal quantum yield of PSII photochemistry; NPQ - nonphotochemical quenching; QV - grapevine cultivar 'Queen of Vineyards'; TAC - total antioxidant capacity; TEAC - Trolox equivalent antioxidant capacity; TPTZ - 2,4,6-tripyridin-2-yl-1,3,5-triazine; Trolox - 6-hydroxy2,5,7,8-tetramethylchroman-2-carboxylic acid; Que-glc - quercetin-3-O-glucoside; Que-gln - quercetin-3-O-glucuronide; UV ultraviolet radiation; UV-A - 315-400 nm UV; UV-B - 280-315 nm UV; WS - grapevine cultivar 'White Sultana'; (NO) $_{(\text {) nonregulated }}$ nonphotochemical quenching; $\mathrm{Y}_{(\mathrm{NPQ})}$ - regulated nonphotochemical quenching; $\Phi_{\mathrm{PSII}}$ - effective quantum yield of PSII photochemistry. Acknowledgements: The project was financed by the National Research, Development and Innovation Office (grant KH-129615 to É. Hideg and K. Csepregi) and by the Higher Education Institutional Excellence Programme of the Ministry of Human Capacities in Hungary, within the framework of the 20765-3/2018/FEKUTSTRAT 'Innovation for sustainable and healthy living and environment' thematic programme of the University of Pécs.

Conflict of interest: The authors declare that they have no conflict of interest. 
that the negative effect may be explained by the oxidation of flavonoids as peroxidase substrates and we also showed that phenolic contents recovered to preirradiation levels within $48 \mathrm{~h}$ (Csepregi et al. 2019). The aim of the present study was to explore whether the metabolic source of this recovery is connected to the berry skin photosynthesis. UV irradiation conditions were the same as in our previous study. However, because it is easier to study white skin berries than dark (red or blue) varieties using chlorophyll fluorescence-based methods, we chose two white table grapes, the thicker-skinned 'Queen of Vineyards' and another cultivar with thinner skin 'White Sultana' (Kriedemann 1968, Crespan et al. 2021). Detached berry clusters were UV-irradiated in the laboratory and changes in berry skin phenolic composition, antioxidant capacity, and photosynthesis were measured at regular intervals after the irradiation. Although grapevine berries as sink organs are assumed to receive metabolites from leaves, they have the capacity to perform photosynthesis, especially during the early stage of fruit development (Blanke and Lenz 1989). The PAM chlorophyll fluorescence method (Schreiber and Bilger 1987) has already been proven useful to measure the photochemical yield of green, immature berry cross-sections (Breia et al. 2013). In the present study, we used mature berries and assessed skin photosynthesis of the whole berries, in comparison to phenolic and antioxidant profiles.

\section{Materials and methods}

Berries and UV treatment: Table grape (Vitis vinifera L.) cultivars 'Queen of Vineyards' and 'White Sultana' were grown in the experimental vineyard of the Research Institute for Viticulture and Oenology, University of Pécs. Ripe grape clusters were harvested and divided into 2-3 parts by cutting the peduncle above rachis internodes. UV irradiation was carried out using the broad band UV-B T-15.M tube of a desktop 15-W UV lamp (VL-215.LM, Vilber-Lourmat, France) as described earlier (Csepregi et al. 2019). After a 30-min irradiation with $0.6 \mathrm{~W} \mathrm{~m}^{-2}$ biologically effective UV-B radiation, berry clusters were stored at $20^{\circ} \mathrm{C}$ under PAR of $50 \mu \mathrm{mol}$ (photon) $\mathrm{m}^{-2} \mathrm{~s}^{-1}$ for $2,8,24,36$, or $48 \mathrm{~h}$. Control berries were stored for either 2 or $48 \mathrm{~h}$ without the UV treatment. After the indicated storage times, berry skin photochemical yields were measured noninvasively (see below), then UV-exposed upper areas of berry skins were removed with a scalpel. For analytical work, skin segments from ten berries were pooled, frozen in liquid nitrogen, and stored at $-80^{\circ} \mathrm{C}$ until lyophilisation. In this way, three samples (originating from ten berries each) were analysed as biological repetitions for each cultivar and storage time. Samples were prepared extracting $60 \mathrm{mg}$ of lyophilized berry skin into $1 \mathrm{~mL}$ of acidified methanol as described earlier (Csepregi et al. 2019).

Chlorophyll (Chl) fluorescence: Following one of the above treatment conditions, berry clusters were kept in darkness for $20 \mathrm{~min}$ before variable $\mathrm{Chl}$ fluorescence parameters were measured with the MAXI version of Imaging-PAM (Heinz Walz GmbH, Effeltrich, Germany). Minimum and maximum $\mathrm{Chl}$ fluorescence yields characteristic to a dark-adapted $\left(\mathrm{F}_{0}\right.$ and $\mathrm{F}_{\mathrm{m}}$, respectively) and a light-acclimated state of PSII ( $\mathrm{F}$ and $\mathrm{F}_{\mathrm{m}}{ }^{\prime}$, respectively) were measured using a saturating pulse (Schreiber et al. 1986). After establishing the maximal quantum yield of PSII photochemistry in dark-adapted samples as $\left(\mathrm{F}_{\mathrm{m}}-\mathrm{F}_{0}\right) / \mathrm{F}_{\mathrm{m}}$, berry clusters were kept under blue actinic light corresponding to PAR of $55 \mu$ mol(photon) $\mathrm{m}^{-2} \mathrm{~s}^{-1}$ for $260 \mathrm{~s}$ in order to achieve the light-adapted state. The $\mathrm{F}$ and $\mathrm{F}_{\mathrm{m}}$ ' fluorescence yields were measured in 20 -s intervals and were used to calculate the following three yield parameters: effective quantum yield of PSII photochemistry $\Phi_{\mathrm{PSII}}=\left(\mathrm{F}_{\mathrm{m}}{ }^{\prime}-\mathrm{F}\right) / \mathrm{F}_{\mathrm{m}}{ }^{\prime}($ Genty et al. 1989), regulated nonphotochemical quenching $\mathrm{Y}_{(\mathrm{NPQ})}=\mathrm{F} / \mathrm{F}_{\mathrm{m}}{ }^{\prime}-$ $\mathrm{F} / \mathrm{F}_{\mathrm{m}}$, and nonregulated nonphotochemical quenching $\mathrm{Y}_{(\mathrm{NO})}=\mathrm{F} / \mathrm{F}_{\mathrm{m}}$ (Klughammer and Schreiber 2008). Using these parameters, NPQ, the total quantum yield of nonphotochemical reactions (Bilger and Björkman 1990), can be calculated as $\mathrm{Y}_{(\mathrm{NPQ})} / \mathrm{Y}_{(\mathrm{NO})}$ (Klughammer and Schreiber 2008). For comparison, three grapevine leaves were also collected from each cultivar and analysed using the above methodology after being stored in darkness for $60 \mathrm{~min}$ while petioles were kept wet in order to avoid water loss.

High-performance liquid chromatography analysis: HPLC analysis was performed on a PerkinElmer Series 200 system (PerkinElmer, Shelton, CT, USA) consisting of a vacuum degassing unit, quaternary pump, autosampler, column thermostat, and a diode-array detector as described earlier (Teszlák et al. 2018). HPLC separations were achieved using a Phenomenex Kinetex $\AA 2.6 \mu \mathrm{m}$ XB-C18 $100 \AA, 100 \times 4.6 \mathrm{~mm}$ column (Phenomenex, Torrance, CA, USA). Gradient elution at $25^{\circ} \mathrm{C}$ was applied using formic acid-acetonitrile mixture with a flow rate of $1 \mathrm{~mL} \mathrm{~min}$. The injection volume of methanolic extracts was $5 \mu \mathrm{L}$. Absorbance of caftaric acid and glycosylated flavonols were monitored at 330 and $350 \mathrm{~nm}$, respectively. For quantification, high purity $(\geq 98 \%)$ external standards (Extrasynthese, Lyon, France) were used. Phenolic compounds of berry skin were expressed as $\mathrm{mg} \mathrm{g}^{-1}(\mathrm{DM})$.

Total antioxidant capacity assays: Berry skin total antioxidant capacities were assessed using two different assays, the Trolox equivalent antioxidant capacity (TEAC) assay and the ferric-reducing antioxidant potential (FRAP) methods. The TEAC assay was carried out according to Re et al. (1999) with modifications (Majer and Hideg 2012). A blue coloured 2,2'-azino-bis-(3-ethylbenzothiazoline6-sulphonic acid) (ABTS) radical solution was prepared by incubating $100 \mu \mathrm{L}$ of ABTS, $100 \mu \mathrm{L}$ of $0.0125 \mathrm{mU}$ horseradish peroxidase, and $100 \mu \mathrm{L}$ of $1 \mathrm{mM} \mathrm{H}_{2} \mathrm{O}_{2}$ in a phosphate buffer $(50 \mathrm{mM}, \mathrm{pH} 6.0)$ for $15 \mathrm{~min}$ at room temperature. Following this, $190 \mu \mathrm{L}$ of ABTS radical solution and $10 \mu \mathrm{L}$ of berry skin extract was mixed in a microplate well and the loss of blue colour was detected at $651 \mathrm{~nm}$ with a plate reader (Multiskan FC, Thermo Fischer Scientific, Walthman, MA, USA). Calibration was 
carried out with 6-hydroxy-2,5,7,8-tetramethylchroman2-carboxylic acid (Trolox) and TEAC data of berry skin extracts were expressed as $\mathrm{mM}$ (Trolox equivalents) $\mathrm{g}^{-1}(\mathrm{DM})$.

The FRAP reagent contained $1.25 \mathrm{~mL}$ of 2,4,6-tripyridin-2-yl-1,3,5-triazine (TPTZ, $10 \mathrm{mM}$ in distilled water), $1.25 \mathrm{~mL}$ of $\mathrm{FeCl}_{3}\left(20 \mathrm{mM} \mathrm{FeCl}_{3}\right.$ in $\left.400 \mathrm{mM} \mathrm{HCl}\right)$, and $12.5 \mathrm{~mL}$ of acetate buffer ( $300 \mathrm{mM}, \mathrm{pH} 3.6)$ (Csepregi and Hideg 2018). Microplate wells were filled with $190 \mu \mathrm{L}$ of this reagent and $10 \mu \mathrm{L}$ of berry skin extract and the reaction mixture was incubated at room temperature for $30 \mathrm{~min}$ before measuring the absorbance at $620 \mathrm{~nm}$ with the plate reader. The FRAP assay was calibrated with ascorbic acid, and data were given as $\mathrm{mM}$ (ascorbic acid equivalents) $\mathrm{g}^{-1}(\mathrm{DM})$.

UV absorbance of berry skin extracts: Berry skin extracts were diluted in ethanol:water: $\mathrm{HCl} \quad(70: 29: 1$, $\mathrm{v}: \mathrm{v}: \mathrm{v})$ and absorption spectra were recorded between 280 and $400 \mathrm{~nm}$ in a 1-cm quartz cuvette using a Shimadzu UV-1800 spectrophotometer (Shimadzu Corporation, Kyoto, Japan). Absorbance was integrated in the UV-A $(315-400 \mathrm{~nm})$ and in the UV-B (280-315 nm) spectral regions.

Statistical analysis: All measured parameters were characterized by means \pm standard deviations (SD). Berry Chl fluorescence measurements were repeated five times for each cultivar and treatment conditions $(n=5)$. Leaf $\mathrm{Chl}$ fluorescence measurements were repeated on three leaves from each cultivar $(n=3)$. Biochemical analyses were performed on three pooled berry skin samples for each cultivar and treatment conditions $(n=3)$. Differences between responses of the two grapevine cultivars to the same treatment were compared by calculating the $P$ probability of the null hypothesis that the two means were equal using two sample Student's $t$-tests. Results of this analysis are shown in figures using one or two asterisks to indicate $0.01<P<0.05$ and $P<0.01$, respectively.
Differences between the responses of the same cultivar to different treatments were analysed using analysis of variance $(A N O V A)$, by calculating the $P$ probability of the null hypothesis that all means were equal. Calculations resulting in $P<0.05$ were followed by post-hoc analyses testing the homogeneity of variances and the normal distribution of residuals using Levene's and SaphiroWilk's tests. Tukey's HSD was used for post-hoc pairwise comparisons of means. Results of the ANOVA tests are shown using different capital letters above columns representing different means in figures. All statistical analyses were carried out using the PAST software (Hammer et al. 2001).

\section{Results and discussion}

\section{Grapevine berries are photochemically active even in their mature state}

Depending on the cultivar, berry skins had 15-20\% lower $\mathrm{F}_{\mathrm{v}} / \mathrm{F}_{\mathrm{m}}$ than that of grapevine leaves but differences in lightacclimated photochemical yields were larger (Fig. 1A). The $42-58 \% \Phi_{\text {PSII }}$ of berry skins as compared to leaves was due to much higher NPQ of the former (Fig. 1B). Fig. 1 also shows that photochemical yields were higher in berries of the 'Queen of Vineyards' (QV) cultivar than that in 'White Sultana' (WS) berries, although the two grapevines were grown at the same location, under identical conditions. There were no significant differences in the $\mathrm{Chl}$ fluorescence-derived parameters of leaves from the two cultivars. Differences between berries and leaves were noticeable not only in steady-state light-acclimated values but also in kinetics. After the onset of actinic light, leaf NPQ undergoes a transient increase before decreasing to a lower steady-state level, in parallel with the rise of $\Phi_{\text {PSII }}$ (Fig. 1A). This change in NPQ is well known and has been explained by a transient, non-assimilatory electron flow, which protects PSII during the first minutes of illumination until the Calvin-Benson cycle is activated (Schreiber and

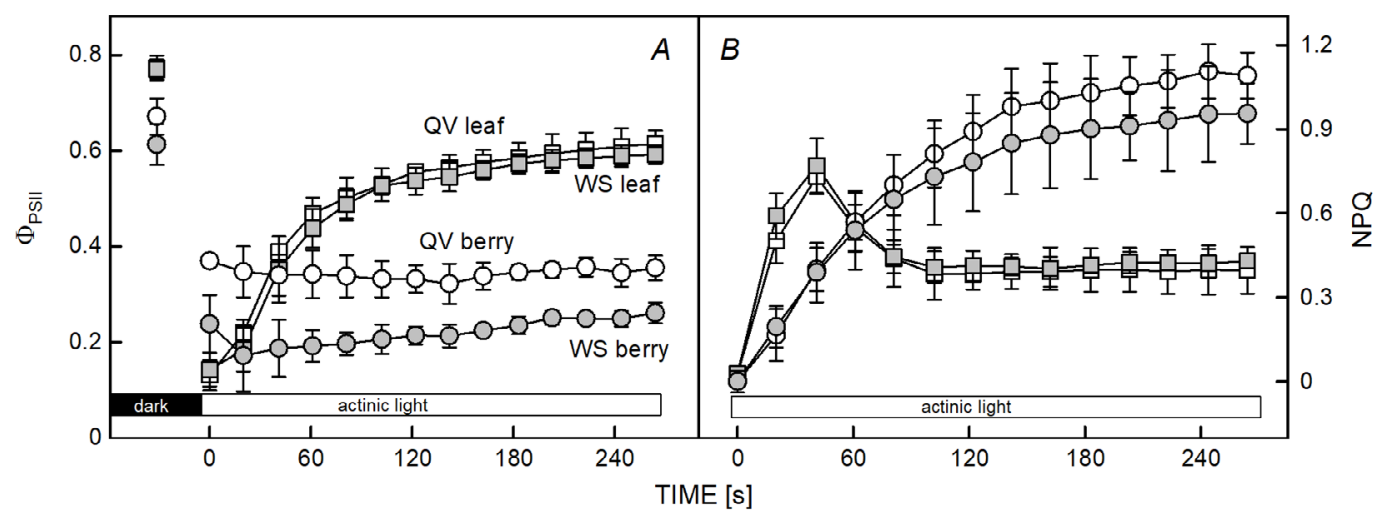

Fig. 1. Changes in photochemical and nonphotochemical yields in response to actinic light $\left[55 \mu\right.$ mol(photon) $\left.\mathrm{m}^{-2} \mathrm{~s}^{-1} \mathrm{PAR}\right] \mathrm{measured}$ in 'Queen of Vineyards' (QV, white symbols) and 'White Sultana' (WS, grey symbols) grapevine berries (circles) and leaves (squares). (A) Maximal quantum yield measured in dark-adapted samples $\left(\mathrm{F}_{\mathrm{v}} / \mathrm{F}_{\mathrm{m}}\right)$ and changes in effective quantum yield measured after the onset of actinic light $\left(\Phi_{\mathrm{PSII}}\right) .(B)$ The build-up of nonphotochemical quenching (NPQ) under actinic light. Symbols and error bars correspond to means and $\operatorname{SD}(n=5$, berries; $n=3$, leaves). 
Bilger 1987, Schreiber and Neubauer 1990). Experiments with heat-exposed tobacco leaves showed that a marked decrease in $\mathrm{CO}_{2}$ fixation removed the second, declining phase of NPQ and resulted in permanent high quenching (Hideg et al. 2008). We propose to explain the observed kinetics of berry skin NPQ build up (Fig. $1 B$ ) by a limited capacity of dark reactions associated with this late developmental phase. The above experiments showed that detached, mature grapevine berries were photochemically active; and may serve as a source of limited, but operational photosynthetic assimilation. Further, we examined the effects of postharvest UV irradiation on berry photochemistry and metabolites. The latter has already been studied in a red grape variety (Csepregi et al. 2019), but the former is shown here for the first time.

\section{Postharvest UV irradiation modifies photochemical and nonphotochemical yields}

Effects of a 30-min irradiation from an UV-B centred, broad-band UV source on berry photosynthesis were measured 2, 8, 24, 36, and $48 \mathrm{~h}$ after the exposure. For reference, untreated berry clusters were also stored for 2 and $48 \mathrm{~h}$. Light-acclimated $\Phi_{\text {PSII }}$ and nonphotochemical quenching were calculated, and the latter was analysed by separating regulated and nonregulated yields $\mathrm{Y}_{(\mathrm{NPQ})}$ and $\mathrm{Y}_{(\mathrm{NO})}$, respectively. This allowed a more direct way to regard possible reasons behind changes in photochemistry than following $\Phi_{\text {PSII }}$ and NPQ only, because the three quantum yields are complementary: $\Phi_{\mathrm{PSII}}+\mathrm{Y}_{(\mathrm{NPQ})}+\mathrm{Y}_{(\mathrm{NO})}=1$ (Klughammer and Schreiber 2008). Fig. 2A,B shows that $\Phi_{\mathrm{PSII}}$ Was lower in WS than that in QV berries throughout

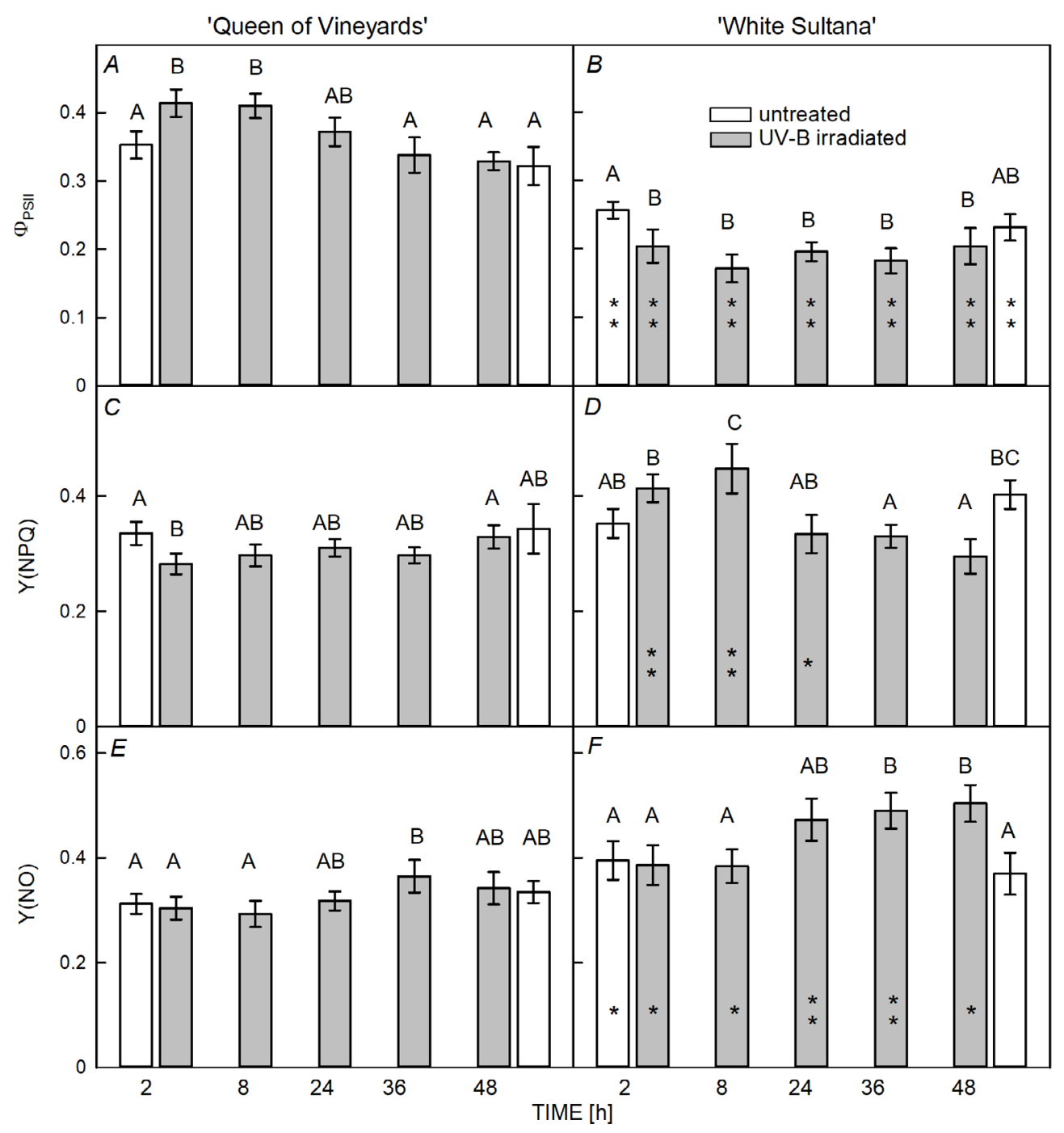

Fig. 2. Light [PAR of $55 \mu \mathrm{mol}\left(\right.$ photon) $\left.\mathrm{m}^{-2} \mathrm{~s}^{-1}\right]$-acclimated photochemical yield $\left(\Phi_{\mathrm{PSII}}\right.$, regulated nonphotochemical quenching [Y $\left.\mathrm{Y}_{(\mathrm{NPQ})}\right]$, and nonregulated nonphotochemical quenching $\left[\mathrm{Y}_{(\mathrm{NO})}\right]$ measured in 'Queen of Vineyards' (left panel) and 'White Sultana' (right panel) grapevine berries. Grey columns represent samples measured at the indicated times after UV irradiation. White columns represent samples stored without irradiation for either 2 or $48 \mathrm{~h}$. Column heights and error bars correspond to means and SD $(n=5)$. Different capital letters above columns represent $P<0.05$ significantly different means measured in the same cultivar. Simple or double asterisks in the right panel represent WS means significantly different from QV means at $P<0.05$ or $P<0.01$, respectively. 
the experiment. Also, the two cultivars responded to the UV treatment differently. Irradiation of QV berries resulted in a 15-20\% increase in photochemistry, which was observed 2 and $8 \mathrm{~h}$ after irradiance. Following this, $\Phi_{\text {PSII }}$ values returned to control levels (Fig. 2A). This was due to a small decrease in regulated nonphotochemical quenching (Fig. 2C), while nonregulated nonphotochemical quenching was unaffected (Fig. 2E), indicating an active downregulation of photoprotective pathways in order to achieve higher electron-transport rates. This change in QV berry photochemistry was not strong, but it may have significant consequences on metabolic responses several hours after the irradiation, as discussed below. Such response to UV irradiation was observed in QV but not in WS berries and the treatment lowered WS berry photochemistry by $20-30 \%$ irreversibly (Fig. $2 B$ ). Decrease in $\Phi_{\text {PSII }}$ was due to increased nonphotochemical quenching, but the loss of photochemical yield to these processes is complex and its nature changed with the time after UV treatment (Fig. 2C,F). First, 2 and $8 \mathrm{~h}$ after irradiation, there was an increase in protective $\mathrm{Y}_{(\mathrm{NPQ})}$ pathways, but this was overridden by higher nonregulated $\mathrm{Y}_{(\mathrm{NO})}$ during subsequent storage indicating long-term stress induced damage to PSII (Kramer et al. 2004, Klughammer and Schreiber 2008).

\section{Postharvest UV irradiation decreases berry skin flavonoid contents}

The phenolic composition of the skin is similar in both white and red grapes apart from the occurrence of anthocyanins in the latter (Braidot et al. 2008). In accordance, the dominant phenolic acid in QV and WS was caftaric acid (CA) (Fig. 3A,B), and major berry skin flavonoids were 3-O-glycosylated flavonols, similarly to the red table grape variety studied earlier (Csepregi et al. 2019). Berry skins

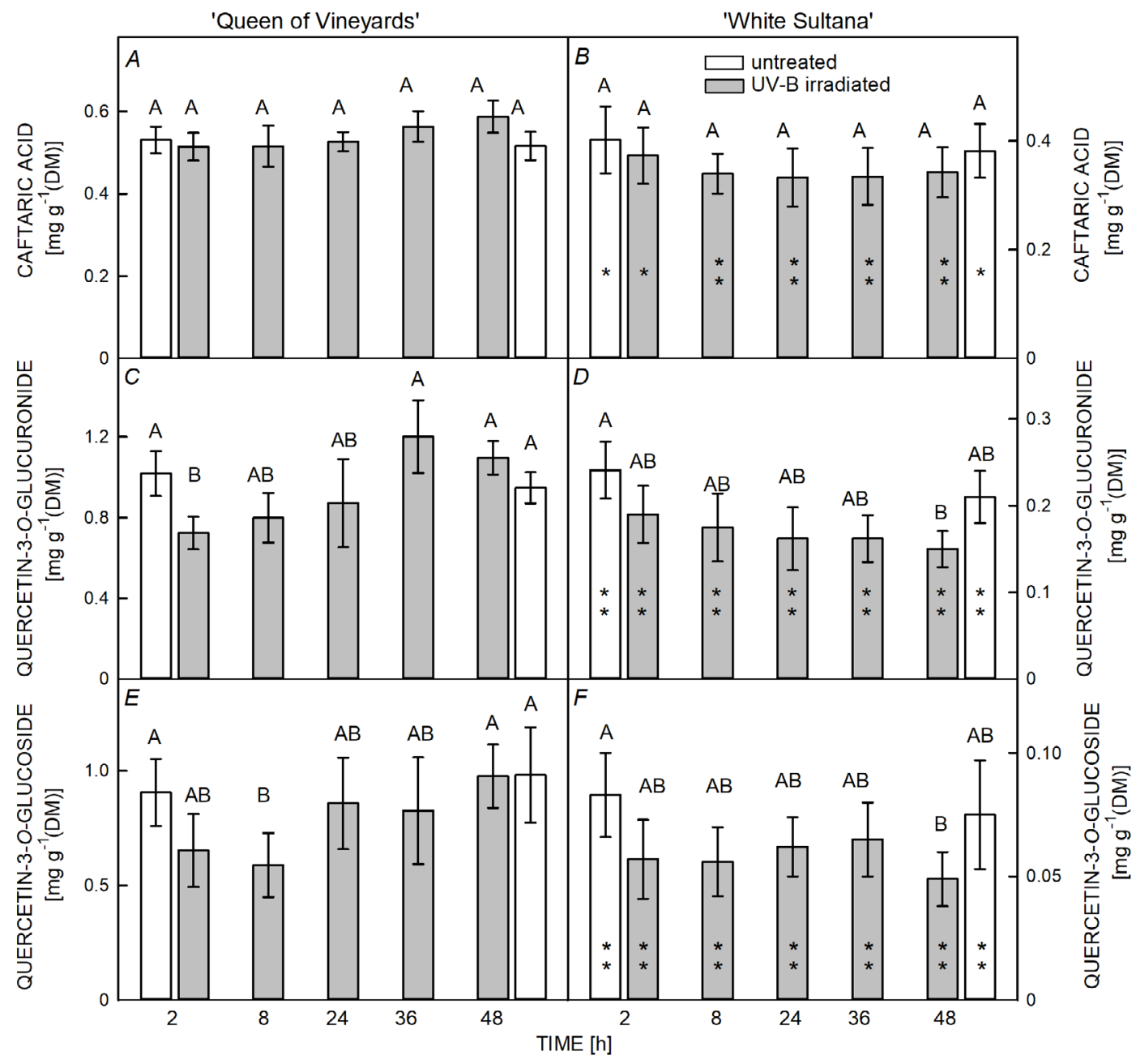

Fig. 3. Contents of major phenolic compounds measured in 'Queen of Vineyards' (left panel) and 'White Sultana' (right panel) grapevine berry skin extracts. Grey columns represent samples measured at the indicated times after UV irradiation. White columns represent samples stored without irradiation for either 2 or $48 \mathrm{~h}$. Column heights and error bars correspond to means and SD $(n=3)$. Different capital letters above columns represent $P<0.05$ significantly different means measured in the same cultivar. Simple or double asterisks in the right panel represent WS means significantly different from QV means at $P<0.05$ or $P<0.01$, respectively. 
from both cultivars contained quercetin-3-O-glucuronide (Que-gln) at the highest, and quercetin-3-O-glucoside (Que-glc) at the second highest concentration (Fig. 3C,F). Quercetin-hyperoside and quercetin-rutinoside were also identified in both cultivars, but at lower concentrations than the above compounds (data not shown). A comparison of the two phenolic profiles identified significantly lower amounts of CA and flavonols in WS than in QV berries, and the difference was more pronounced in the amounts of flavonols than in CA (Fig. 3). Although UV-induced changes in mean concentrations suggest tendencies, high biological variation masked significant effects in most cases. Significant changes were, however, observed in the Que-gln and Que-glc contents of QV berries, which decreased 2 and $8 \mathrm{~h}$ after the UV treatment, but recovered to control levels during a longer storage (Fig. 3C). Untreated berry skins of the WS cultivar contained $80 \%$ less Que-gln than that of QV samples and the postharvest UV treatment decreased this concentration further, by approximately $30 \%$ as measured $48 \mathrm{~h}$ after the irradiation. In plant tissues, flavonoid contents may decrease as a result of oxidation, either in a direct reaction with reactive oxygen species (ROS) and other free radicals, or during electron donation to phenolic peroxidases. Both reactions are relevant to the present study because glycosylated quercetins are strong antioxidants (Csepregi et al. 2016, Csepregi and Hideg 2018) and some may also serve as peroxidase substrates (Rácz et al. 2020). In an earlier experiment, using the same UV irradiation but a different cultivar than that in the present study, postharvest UV increased berry skin peroxidase activity after $2 \mathrm{~h}$ but not 24 h later (Csepregi et al. 2019). A similar mechanism, a temporary increase in berry skin peroxidase activities, may be the explanation for the observed decrease in QV and WS flavonoids (Fig. 3). Recovery in QV but not in WS may be related to a higher photosynthetic activity, facilitating a more efficient synthesis and replacement of oxidised flavonoids in QV than that in WS.

\section{Postharvest UV irradiation decreases berry skin antioxidant contents}

The two total antioxidants capacity assays applied in this study reflect general, electron-donating antioxidant capacities of all extractable compounds (Huang et al. 2005), although different phenolic compound groups have different reactivities to the chromophores applied in the assays (Csepregi et al. 2016). Both methods confirmed that QV samples had higher antioxidant capacities than that of WS ones (Fig. 4) and that the UV treatment permanently reduced the amounts of antioxidants in the latter (Fig. 4B,D). Significant UV-induced changes in QV antioxidants were registered with the TEAC but not with the FRAP method, which can be explained by the stronger affinity of the less affected compound, CA, to FRAP than to TEAC (Csepregi et al. 2016). Similarities between changes in antioxidant capacities and flavonoid content in QV samples during the 48-h period after the UV

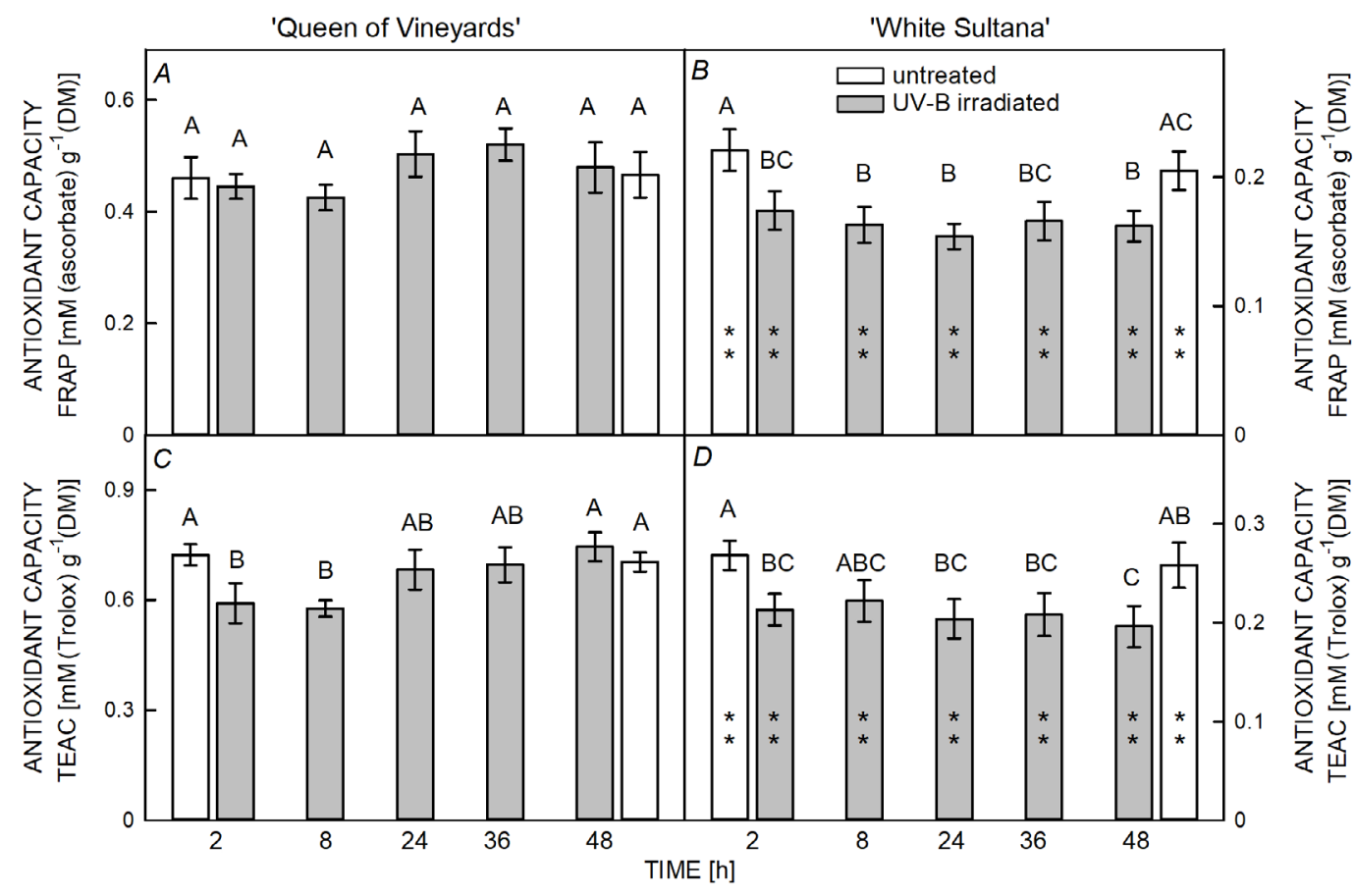

Fig. 4. Total antioxidant capacities measured in 'Queen of Vineyards' (left panel) and 'White Sultana' (right panel) grapevine berry skin extracts. Grey columns represent samples measured at the indicated times after UV irradiation. White columns represent samples stored without irradiation for either 2 or $48 \mathrm{~h}$. Column heights and error bars correspond to means and SD $(n=3)$. Different capital letters above columns represent $P<0.05$ significantly different means measured in the same cultivar. Simple or double asterisks in the right panel represent WS means significantly different from QV means at $P<0.05$ or $P<0.01$, respectively. 
treatment suggest that Que-gln or Que-glc may also act as direct antioxidants in the berries, although there is no direct proof to support this hypothesis so far.

In addition to lower base antioxidant concentrations, reasons behind the observed different sensitivities of QV and WS berries to postharvest UV treatment may also include a more efficient penetration of both UV-B and $\mathrm{UV}-\mathrm{A}$ radiation into berry skins in the WS than in the QV cultivar. In addition to the known difference in skin thickness (Reynolds 2015), the spectrophotometry of skin extracts identified that QV samples had approximately 2- and 3-fold higher UV-B and UV-A absorbance, respectively, than that of WS samples (data not shown). Although tissue optics may also affect UV absorption in situ, absorbance values of skin extracts suggest that radiation from the applied broad band (UV-B + UV-A) source penetrated deeper into WS than QV berries. This would explain the observed stronger effect on photosynthesis and the impaired ability recovery of WS berries after the UV treatment.

It is important to note that in the above study, chlorophyll fluorescence-derived yield parameters measurements were carried out under relatively low PAR. Under 600-1,200 $\mu \mathrm{mol}$ (photon) $\mathrm{m}^{-2} \mathrm{~s}^{-1}$ corresponding to photon flux densities outdoors at harvest time, photochemical yields of mature berries were lower and more absorbed quanta were lost to nonphotochemical quenching (data not shown). In this way, our findings are relevant to storage conditions, and demonstrate - to our best knowledge for the first time - that mature berry skins may have active photosynthesis supporting postharvest metabolic adjustments.

\section{References}

Bilger W., Björkman O.: Role of the xanthophyll cycle in photoprotection elucidated by measurements of light-induced absorbance changes, fluorescence and photosynthesis in leaves of Hedera canariensis. - Photosynth. Res. 25: 173186,1990

Blanke M.M., Lenz F.: Fruit photosynthesis. - Plant Cell Environ. 12: 31-46, 1989.

Braidot E., Zancani M., Petrussa E. et al.: Transport and accumulation of flavonoids in grapevine (Vitis vinifera L.). Plant Signal. Behav. 3: 626-632, 2008.

Breia R., Vieira S., da Silva J.M. et al.: Mapping grape berry photosynthesis by chlorophyll fluorescence imaging: the effect of saturating pulse intensity in different tissues. Photochem. Photobiol. 89: 579-585, 2013.

Cantos E., García-Viguera C., de Pascual-Teresa S., TomásBarberán F.A.: Effect of postharvest ultraviolet irradiation on resveratrol and other phenolics of $\mathrm{cv}$. Napoleon table grapes. - J. Agr. Food Chem. 48: 4606-4612, 2000.

Carbonell-Bejerano P., Diago M.-P., Martínez-Abaigar J. et al.: Solar ultraviolet radiation is necessary to enhance grapevine fruit ripening transcriptional and phenolic responses. - BMC Plant Biol. 14: 183, 2014.

Conde C., Silva P., Fontes N. et al.: Biochemical changes throughout grape berry development and fruit and wine quality. - Food 1: 1-22, 2007.

Crespan M., Migliaro D., Vezzulli S. et al.: A major QTL is associated with berry grape texture characteristics. - OENO
One 55: 183-206, 2021.

Csepregi K., Hideg É.: Phenolic compound diversity explored in the context of photo-oxidative stress protection. - Phytochem. Anal. 29: 129-136, 2018.

Csepregi K., Körösi L., Teszlák P., Hideg É.: Postharvest UV-A and UV-B treatments may cause a transient decrease in grape berry skin flavonol-glycoside contents and total antioxidant capacities. - Phytochem. Lett. 31: 63-68, 2019.

Csepregi K., Neugart S., Schreiner M., Hideg É.: Comparative evaluation of total antioxidant capacities of plant polyphenols. - Molecules 21: 208, 2016.

Genty B., Briantais J.-M., Baker N.R.: The relationship between the quantum yield of photosynthetic electron transport and quenching of chlorophyll fluorescence. - BBA-Gen. Subjects 990: 87-92, 1989

Graf B.A., Milbury P.E., Blumberg J.B.: Flavonols, flavones, flavanones, and human health: Epidemiological evidence. J. Med. Food 8: 281-290, 2005.

Hammer Ø., Harper D.A.T., Ryan P.D.: PAST: Paleontological statistics software package for education and data analysis. Palaeontol. Electron. 4: 1-9, 2001.

Hideg É., Kós B.P., Schreiber U.: Imaging of NPQ and ROS formation in tobacco leaves: heat inactivation of the waterwater cycle prevents down-regulation of PSII. - Plant Cell Physiol. 42: 1879-1886, 2008.

Huang D., Ou B., Prior R.L.: The chemistry behind antioxidant capacity assays. - J. Agr. Food Chem. 53: 1841-1856, 2005.

Klughammer C., Schreiber U.: Complementary PSII quantum yields calculated from simple fluorescence parameters measured by PAM fluorometry and the saturation pulse method. - PAM Appl. Notes 1: 27-35, 2008.

Kramer D.M., Johnson G., Kiirats O., Edwards G.E.: New fluorescence parameters for the determination of $\mathrm{Q}_{\mathrm{A}}$ redox state and excitation energy fluxes. - Photosynth. Res. 79: 209-218, 2004.

Kriedemann P.E.: Observations on gas exchange in the developing sultana berry. - Aust. J. Biol. Sci. 21: 907-916, 1968.

Majer P., Hideg É.: Developmental stage is an important factor that determines the antioxidant responses of young and old grapevine leaves under UV irradiation in a green-house. Plant Physiol. Bioch. 50: 15-23, 2012.

Rácz A., Czégény Gy., Csepregi K., Hideg É.: Ultraviolet-B acclimation is supported by functionally heterogeneous phenolic peroxidases. - Sci. Rep.-UK 10: 16303, 2020.

Re R., Pellegrini N., Proteggente A. et al.: Antioxidant activity applying an improved ABTS radical cation decolorization assay. - Free Radical Bio. Med. 26: 1231-1237, 1999.

Reynolds A.: Grapevine Breeding Programs for the Wine Industry. Woodhead Publishing Series in Food Science, Technology and Nutrition. Pp. 439. Elsevier, Cambridge 2015.

Schreiber U., Bilger W.: Rapid assessment of stress effects on plant leaves by chlorophyll fluorescence measurements. - In: Tenhunen J.D., Catarino F.M., Lange O.L., Oechel W.C. (ed.): Plant Response to Stress. NATO ASI Series. Vol. 15. Pp. $27-$ 53. Springer, Berlin-Heidelberg 1987.

SchreiberU., Neubauer C.: $\mathrm{O}_{2}$-dependent electron flow, membrane energization and the mechanism of nonphotochemical quenching of chlorophyll fluorescence. - Photosynth. Res. 25: 279-293, 1990.

Schreiber U., Schliwa U., Bilger W.: Continuous recording of photochemical and non-photochemical chlorophyll fluorescence quenching with a new type of modulation fluorometer. - Photosynth. Res. 10: 51-62, 1986.

Sheng K., Zheng H., Shui S. et al.: Comparison of postharvest UV-B and UV-C treatments on table grape: Changes in phenolic compounds and their transcription of biosynthetic 
genes during storage. - Postharvest Biol. Tec. 138: 74-81, 2018 .

Teszlák P., Kocsis M., Scarpellini A. et al.: Foliar exposure of grapevine (Vitis vinifera $\mathrm{L}$.) to $\mathrm{TiO}_{2}$ nanoparticles under field conditions: Photosynthetic response and flavonol profile. Photosynthetica 56: 1378-1386, 2018.

(C) The authors. This is an open access article distributed under the terms of the Creative Commons BY-NC-ND Licence. 